From plane waves to local Gaussians for the simulation of correlated periodic systems

George H. Booth' , Theodoros Tsatsoulis, Garnet Kin-Lic Chan, and Andreas Grüneis'

Citation: J. Chem. Phys. 145, 084111 (2016); doi: 10.1063/1.4961301

View online: http://dx.doi.org/10.1063/1.4961301

View Table of Contents: http://aip.scitation.org/toc/jcp/145/8

Published by the American Institute of Physics 


\title{
From plane waves to local Gaussians for the simulation of correlated periodic systems
}

\author{
George H. Booth, ${ }^{1, a)}$ Theodoros Tsatsoulis, ${ }^{2}$ Garnet Kin-Lic Chan, ${ }^{3}$ \\ and Andreas Grüneis ${ }^{2, b)}$ \\ ${ }^{1}$ Department of Physics, King's College London, Strand, London WC2R 2LS, United Kingdom \\ ${ }^{2}$ Max Planck Institute for Solid State Research, Heisenbergstraße 1, 70569 Stuttgart, Germany \\ ${ }^{3}$ Frick Laboratory, Department of Chemistry, Princeton University, Princeton, New Jersey 08544, USA
}

(Received 21 March 2016; accepted 7 August 2016; published online 29 August 2016)

\begin{abstract}
We present a simple, robust, and black-box approach to the implementation and use of local, periodic, atom-centered Gaussian basis functions within a plane wave code, in a computationally efficient manner. The procedure outlined is based on the representation of the Gaussians within a finite bandwidth by their underlying plane wave coefficients. The core region is handled within the projected augment wave framework, by pseudizing the Gaussian functions within a cutoff radius around each nucleus, smoothing the functions so that they are faithfully represented by a plane wave basis with only moderate kinetic energy cutoff. To mitigate the effects of the basis set superposition error and incompleteness at the mean-field level introduced by the Gaussian basis, we also propose a hybrid approach, whereby the complete occupied space is first converged within a large plane wave basis, and the Gaussian basis used to construct a complementary virtual space for the application of correlated methods. We demonstrate that these pseudized Gaussians yield compact and systematically improvable spaces with an accuracy comparable to their non-pseudized Gaussian counterparts. A key advantage of the described method is its ability to efficiently capture and describe electronic correlation effects of weakly bound and low-dimensional systems, where plane waves are not sufficiently compact or able to be truncated without unphysical artifacts. We investigate the accuracy of the pseudized Gaussians for the water dimer interaction, neon solid, and water adsorption on a LiH surface, at the level of second-order Møller-Plesset perturbation theory. Published by AIP Publishing. [http://dx.doi.org/10.1063/1.4961301]
\end{abstract}

\section{INTRODUCTION}

In the development of first principles electronic structure methods for extended systems, a huge amount of research effort is expended exploring different function spaces to expand the electronic wavefunctions. This is a critical design choice since the rate of convergence of the desired properties of the system with respect to the size of this function space will substantially impact the computational cost and feasibility of calculations, and go a long way to determining the utility of the approach. In this paper, we will consider a simple, robust protocol for building a periodic Gaussian basis from an underlying traditional plane-wave expansion. In particular, we focus on the ability to converge the virtual manifold of states, required for correlated, post-mean-field calculations, such as the Random Phase Approximation (RPA), GW theory, MøllerPlesset theory (MP2), coupled-cluster theory, and multiconfigurational strong correlation approaches. ${ }^{1}$ The computational cost of these methods grows between quadratically and exponentially with respect to the number of virtual states, and therefore the ability to span the relevant parts of this space with as few functions as possible becomes a critical design decision in the implementation of periodic correlated methods.

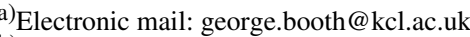

b)Electronic mail: a.grueneis@ @ fkf.mpg.de
}

From the perspective of the paradigmatic uniform electron gas, plane waves are the natural choice of basis to expand both one-electron and many-electron wavefunction quantities. $^{2-5}$ These plane waves are eigenfunctions of the kinetic energy operator, and naturally fulfil the periodicity of the computational cell. In realistic ab initio calculations, these plane waves also have a number of appealing features. They are independent of the molecular makeup of the simulation cell, and instead only depend on the size and geometry of the cell. In addition, a single cutoff parameter dictating the upper energy scale of the included plane waves (and hence the resolution of the resultant wavefunctions) is used to systematically expand the plane wave basis to completeness, in a fashion free from basis-set superposition error (BSSE). ${ }^{6,7}$

However, there are some drawbacks to plane wave expansions. Since they make no reference to the nature of the atomic environment, they have equal basis coverage throughout the cell. This can lead to a great deal of wasted computational effort when studying defects, surfaces or lowerdimensional systems. This is due to the necessity for large amounts of vacuum in the cells to minimize the effect of spurious periodic images, which results in large, unwieldy plane wave expansions to converge relevant properties. ${ }^{8}$ Even in bulk systems, the dominant electron density will generally be clustered around the atomic sites, and making use of this 
fact with atom-centered functions can certainly improve basis set convergence.

For correlated methods, the steeper scaling compared to mean-field methods means that the speed of convergence with respect to number of basis functions is even more critical. Rather than using plane waves directly for the virtual space, it is more common to truncate the prior mean-field virtual manifold on an energetic criteria to improve the convergence to the complete basis set. ${ }^{9,10}$ However, this virtual manifold is not inherently physical, and its somewhat arbitrary truncation therefore does not necessarily provide a good basis to expand the correlated wavefunction. Truncating based on an energetic criteria (now on the mean-field energy rather than kinetic energy as for plane waves) does not necessarily yield fast convergence, while issues such as band-crossings which can occur as unit cells are distorted or enlarged can yield discontinuities in potential energy surfaces and equations of state. Furthermore, consistent truncations of the virtual bandstructure when comparing fundamentally different systems, such as a defective and pristine lattice structure, are close to impossible to achieve, and therefore still generally rely on convergence to costly, near-complete basis sets for meaningful comparisons. Attempts to truncate virtual single-particle orbital expansions using other criteria, such as occupation numbers from other levels of theory, have had some success, but can often be expensive to carry out. ${ }^{8}$

An alternative representation to plane wave expansions is local atom-centered basis sets. This mirrors the duality between basis representations of lattice models such as the Hubbard model, where the local "site" degrees of freedom contrast with that of the discrete $k$-space, plane wave representation, commonly used in the uniform electron gas. These local functions now correspond to a basis with a local, atomistic description of the simulation cell. Furthermore, their use allows for simple extraction of local descriptors, such as atomic electron numbers, spin density, or projected densities of states, without requiring post-processing localization steps towards Wannier, or similar, functions. ${ }^{11,12}$ While these local functions can take many forms, from Muffin-tin orbitals ${ }^{13-15}$ to wavelets ${ }^{16-18}$ or numerical atomic orbitals, ${ }^{19,20}$ in this manuscript we consider the use of periodic Gaussian basis sets and analyse their convergence for correlated levels of theory.

While Gaussian basis sets can potentially take many different parameterizations, their widespread use within the field of quantum chemistry has meant that many tabulated basis sets of increasing size and flexibility are readily available. ${ }^{21,22}$ The Gaussian orbitals are optimized to approximate the natural orbitals of the free atom and its common ions, often at correlated levels of theory. Orbitals beyond the core and valence shells are included to account for appropriate polarization and distortion of the atomic wavefunctions in bonding environments, and to provide a description of correlation effects. Basis sets are commonly arranged in hierarchies so that they can be systematically expanded to allow for consistent and (if necessary) extrapolatable convergence. In periodic systems, as the atomic-like Gaussian orbitals come together to form bands, they will split about the Fermi level to describe the important low energy regions of the space, as well as retaining a consistent, local description of the one-electron wavefunctions, even for low-dimensional systems, or as cells change shape or atoms move. The use of Gaussian-type orbitals (GTOs) in periodic electronic structure is not new to this work, with several other codes employing their use. ${ }^{23-31}$ The local nature of these functions and "nearsightedness" of the interactions is often used for reduced scaling techniques, including in diagonalization steps or construction of Coulomb and exchange interactions in order to approach linear scaling mean-field treatments, ${ }^{32-35}$ and can also be extended to local treatment of correlation. ${ }^{25,36}$ Furthermore, mixed plane-wave and Gaussian schemes have also been previously introduced as an attempt to combine their strengths in the condensed phase. $^{37}$

It should be noted that in post-mean-field correlated methods (including those based around the explicitly screened Coulomb interaction) the ultimate rate determining scaling in the convergence of correlated properties with respect to the one-electron basis set size is the description of the shortrange Coulomb hole and non-analytic cusp condition at the coalescence point of two particles. ${ }^{38,39}$ It has been shown that this scaling behaviour is the same for both Gaussian and plane wave expansions of the orbital space. ${ }^{2,39}$ However, the absolute convergence in different basis sets can be very different, as the decay of the Coulomb hole depends sensitively on the electron density, as well as the flexibility of the basis at the coalescence points. Furthermore, the absolute one-electron basis set incompleteness (which is both a feature of the correlated and mean-field wavefunctions) is also very much dependent on the specifics of the primitive orbital expansion. ${ }^{40}$

In this paper, we detail an implementation of a straightforward approach for the use of a periodic Gaussian basis (or indeed any numerical atom-centered functions) within a code set up for more traditional plane wave description of the wavefunction. We also consider changes to deal with core electrons when they are not explicitly considered, as is the case in the VASP code within the Projector Augmented Wave (PAW) framework where this work is implemented. We then apply correlated levels of theory to a number of systems, demonstrating that the consistent level of truncation as afforded by the Gaussian basis set expansions leads to a rapidly convergent and extrapolatable virtual space for the calculations. Extensive comparison is made to allelectron molecular calculations, giving confidence in the applicability of the functions for both strongly and weakly interacting systems.

For larger-scale applications, we propose and explore an efficient hybrid approach. In this approach, the occupied orbitals are converged first within a large primitive plane wave expansion, rendering the occupied space and hence Hartree-Fock energy and its contributions to properties essentially complete. A virtual basis is then included for the correlation treatment comprised of the complementary set of orbitals constructed by projecting the occupied orbitals out of the Gaussian basis set. This dual approach removes basis incompleteness of the occupied one-electron wavefunctions and properties and thus substantially ameliorates the issue of basis-set superposition error, which now only manifests through the subsequent correlation treatment. In addition, 
it retains the benefits of the compact, consistent virtual space afforded by the atom-centered Gaussians. We note that related occupied projected Gaussian bases (projected atomic orbitals ${ }^{41}$ ) have been used previously in Gaussian basis codes to exploit the locality of correlation: ${ }^{24,36}$ however, we do not rely on this locality here, beyond its manifestation in the general compactness of the full set of virtual orbitals, as discussed above. This is applied to the MP2 contributions to the cohesive energy of the neon solid, where the weak binding means that basis set incompleteness manifests as large relative errors. Also studied is the absorption of a water molecule onto a lithium hydride crystal surface, where the low-dimensionality of the system means that the Gaussian virtual space efficiently spans the correlated wavefunction and allows for rapid convergence of extrapolations which agree well with reference results.

\section{CONSTRUCTION OF GAUSSIAN BASIS}

This section outlines the procedures employed to transform a specified contracted Gaussian basis set into one which can be used with a plane wave solid state code within the framework of the projector augmented wave method. This involves first "pseudizing" the sharply peaked core part of the basis, designed to capture the nuclear cusp within all-electron calculations, but which is unnecessary in this context. The resulting smoother function is then represented through its plane wave coefficients. Figure 1 outlines the individual steps schematically.

\section{A. Gaussian basis functions}

The Gaussian basis set is composed of atom-centered functions (GTOs) that can be decomposed into radial and angular parts such that an angular momentum function $(m, l)$ for a given atom positioned at $\mathbf{R}$ can be given by

$$
G_{m, l, \mathbf{R}}(\mathbf{r})=R_{l}(|\mathbf{r}-\mathbf{R}|) Y_{l, m}(\Theta, \phi),
$$

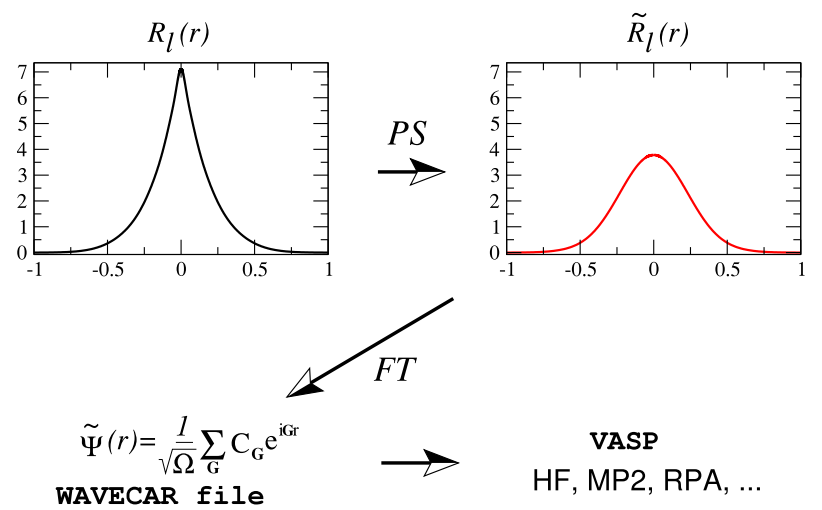

FIG. 1. Schematic illustration of the workflow. A Gaussian basis set is represented on a radial grid (left upper panel). We employ a pseudization strategy (PS) to allow the core to be more efficiently represented by its Fourier coefficients by calculating the corresponding pseudized basis function (right upper panel). In the following step we Fourier transform (FT) the pseudized Gaussian-type orbital (PGTO) to the plane wave basis (left bottom panel). This is then used for the various electronic structure methods ranging from Hartree-Fock (HF) to post-HF theories. where $Y_{l m}(\Theta, \phi)$ is a spherical harmonic and $\mathbf{r}, \Theta, \phi$ correspond to spherical coordinates. The radial function $R_{l}(|\mathbf{r}-\mathbf{R}|)$ is expanded using Gaussian functions such that

$$
R_{l}(|\mathbf{r}-\mathbf{R}|)=|\mathbf{r}-\mathbf{R}|^{l} \sum_{p} c_{p} A\left(l, \alpha_{p}\right) e^{-\alpha_{p}|\mathbf{r}-\mathbf{R}|^{2}},
$$

where $A\left(l, \alpha_{p}\right)$ is a normalization constant of the Gaussian primitives. $c_{p}$ are contraction coefficients for the primitive Gaussian functions with exponent $\alpha_{p}$.

\section{B. The PAW method}

The projector augmented wave (PAW) method was introduced by Blöchl. ${ }^{42}$ Further details, as well as its close relationship with the ultrasoft pseudopotential method of Vanderbilt, were shown by Kresse and Joubert in Ref. 43, while here we briefly recap the approach. In the PAW method, the orbitals $\left(\left|\psi_{n}\right\rangle\right)$ are derived from the pseudo-orbitals $\left(\left|\tilde{\psi}_{n}\right\rangle\right)$ by means of a linear transformation

$$
\left|\psi_{n}\right\rangle=\left|\tilde{\psi}_{n}\right\rangle+\sum_{i}\left(\left|\varphi_{i}\right\rangle-\left|\tilde{\varphi}_{i}\right\rangle\right)\left\langle\tilde{p}_{i} \mid \tilde{\psi}_{n}\right\rangle .
$$

The index $n$, labeling the orbitals $\psi$, is understood to be shorthand for the band index and the Bloch wave vector $k_{n}$, while the index $i$ is a shorthand for the atomic site $R_{i}$, the angular momentum quantum numbers $l_{i}$ and $m_{i}$, and an additional index $\epsilon_{i}$ denoting the linearization energy. The wave vector is conventionally chosen to lie within the first Brillouin zone. The pseudo-orbitals are the variational quantities of the PAW method and are expanded in reciprocal space using plane waves,

$$
\left\langle\mathbf{r} \mid \tilde{\Psi}_{n}\right\rangle=\frac{1}{\sqrt{\Omega}} \sum_{\mathbf{G}} C_{\mathbf{G}}^{n} e^{i\left(k_{n}+\mathbf{G}\right) \mathbf{r}} .
$$

The all-electron partial waves $\varphi_{i}$ are the solution to the radial Schrödinger equation for the non-spin-polarized reference atom at specific energies $\epsilon_{i}$ and specific angular momenta $l_{i}$. The pseudo-partial waves $\tilde{\varphi}_{i}$ are equivalent to the all-electron partial waves outside a core radius $r_{c}$ and match continuously onto $\varphi_{i}$ inside the core radius. The partial waves $\varphi_{i}$ and $\tilde{\varphi}_{i}$ are represented on radial logarithmic grids, multiplied with spherical harmonics. The projector functions $\tilde{p}_{i}$ are constructed in such a way that they are dual to the pseudo-partial waves, i.e., $\left\langle\tilde{p}_{i} \mid \tilde{\varphi}_{j}\right\rangle=\delta_{i j}$. The pseudized partial waves $\tilde{\varphi}_{i}$ are obtained by pseudizing the all-electron partial waves $\varphi_{i}$ for a given core radius $r_{c} \cdot{ }^{44}$

\section{Pseudized Gaussians}

In this work we seek to employ Gaussian basis sets using a plane wave code. Fourier components of strongly localized real space orbitals decay very slowly, which manifests as a slow convergence of the orbitals with respect to the underlying plane wave energy cutoff. This cutoff energy dictates the size of the plane wave basis employed in Eq. (4). The slow convergence of the orbitals with respect to this cutoff is mainly due to sharp features of the Gaussian-type orbitals (GTOs) resulting from the fitting of the non-analytic cusp behaviour at the nuclear coalescence point, which in non-relativistic 
quantum theory exhibits a derivative discontinuity in the wavefunction. However, the plane-wave basis we employ is augmented within the projector augmented wave framework, which includes a description of the atomic core region of each atom. This augmentation largely resolves these sharply varying parts of the wavefunction.

To this end we "pseudize" the GTO functions defined in Eq. (2), which smooths the core region of each function, defined up to a pseudization radius from the nucleus, $r_{c}$. This is done in a way consistent with the symmetry and norm of the orbitals, and results in a more rapidly convergent set of Fourier components representing pseudized GTOs (PGTOs). The employed pseudization strategy mirrors the work of Kresse et al. in the construction of pseudized partial waves ${ }^{44}$ for pseudopotentials. The core of the pseudized radial Gaussian basis functions is expanded in three spherical Bessel functions such that

$$
\tilde{R}_{l}(r)=\sum_{i=1}^{3} \alpha_{i} r j_{l}\left(q_{i} r\right),
$$

with $q_{i}$ chosen such that the value of the function as well as logarithmic derivatives matches at the cutoff radius,

$$
\left.\frac{\partial}{\partial r}\left[\ln R_{l}(r)\right]\right|_{r=r_{c}}=\left.\frac{\partial}{\partial r}\left[\ln \left(r j_{l}\left(q_{i} r\right)\right)\right]\right|_{r=r_{C}} .
$$

Moreover, we require norm conservation of the PGTO such that

$$
\int_{0}^{r_{c}} \tilde{R}_{l}(r)^{2} d r=\int_{0}^{r_{c}} R_{l}(r)^{2} d r .
$$

We note that for $r \geq r_{c}$ the following condition holds $R_{l}(r)=\tilde{R}_{l}(r)$. We choose the pseudization radius such that it is identical to the cutoff radius used by the projectors $\tilde{p}_{i}$ in the PAW method. In this manner we ensure that core region of the pseudized Gaussians is augmented with additional terms that capture the oscillatory and sharp features of the one-electron wavefunctions in this region. Once the appropriate Fourier components are found, integrals between the orbitals can be obtained in the reciprocal basis as normal, with the Gygi-Baldereschi scheme used to correct for the divergence at $\mathbf{G}=0$ of the Coulomb kernel in reciprocal space. $^{4,45}$

A demonstration of the importance of the pseudization of the atomic Gaussian functions is given in Fig. 2, where the convergence of the total and correlation energies of a $3 \times 3 \times 3$ cell of diamond is considered, demonstrating that pseudization of the atomic functions is essential to obtain rapidly convergent properties with the size of the underlying plane wave basis. While the size of this plane wave basis is generally insignificant when considering the cost of the correlated treatment in the system, numerical and computational difficulties can arise if the plane wave cutoff is too large, and therefore the pseudization is necessary when aiming to converge results for a given atomic basis set. We can also consider the convergence of static properties in a larger Gaussian basis as the underlying plane wave basis which represents these functions increase, which will depend on the accuracy of relative energies across a range of cell geometries. This is shown in

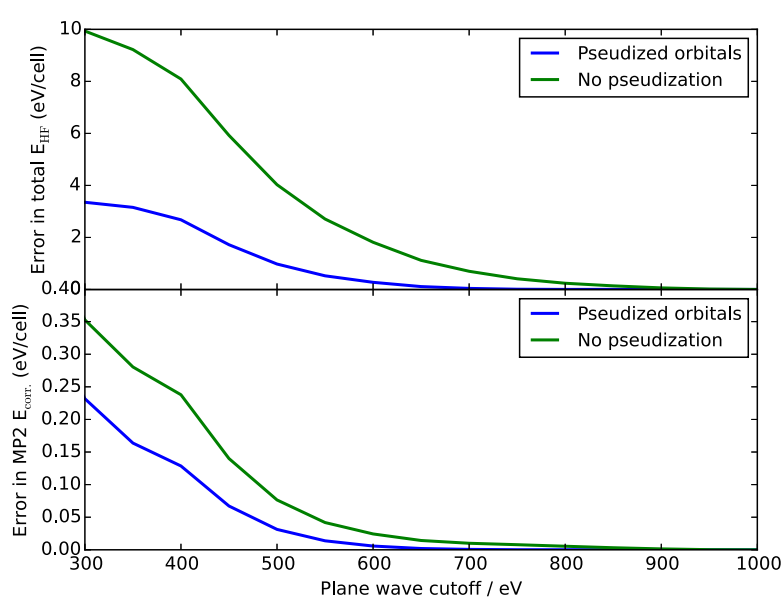

FIG. 2. The effect of pseudization on the convergence of Gaussian orbitals as plane waves, for a $3 \times 3 \times 3$ simulation cell of diamond. The upper plot shows the difference between the Hartree-Fock total energy for the pseudized and non-pseudized STO-3G Gaussian orbital basis. This is calculated as the difference between a plane wave expansion of the orbitals truncated at a given energy and the "complete" (1000 eV cutoff) description of the same orbital space. Note that the $1000 \mathrm{eV}$ cutoff energies are not the same, since the act of pseudization slightly alters the orbitals. The lower plot shows the convergence of the MP2 correlation energy for the same system. The 1s orbitals are removed from the STO-3G atomic basis on each atom, since the $1 \mathrm{~s}$ electrons are replaced by a pseudopotential, with the orbitals represented within the PAW functions.

Fig. 3, demonstrating that cutoffs of $750 \mathrm{eV}$ are sufficient to saturate the representation of the pseudized Gaussian orbitals, and to fully converge the equation of state for the system.

\section{Virtual pseudized Gaussian basis}

In instances where the local nature of the basis as Gaussians is not specifically required in the mean-field calculation, it can be highly beneficial to pursue a hybrid

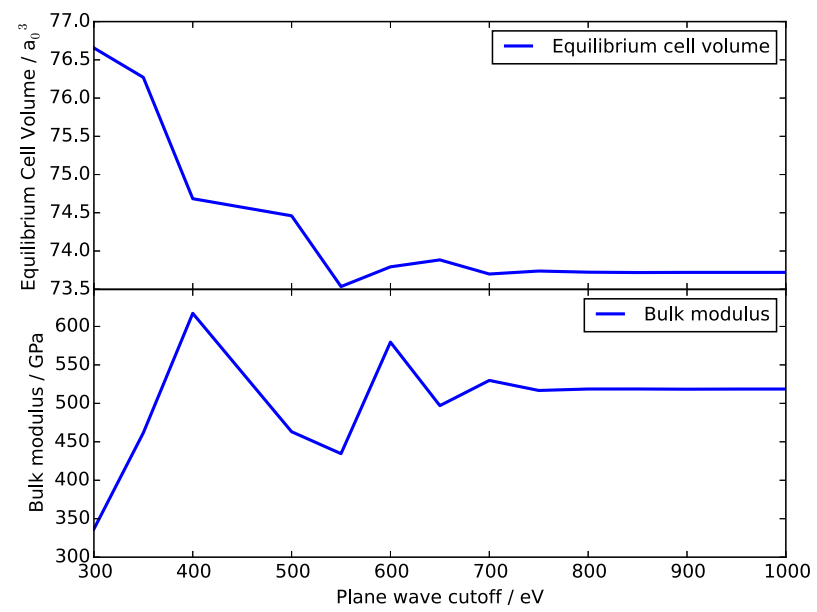

FIG. 3. Convergence of the equilibrium cell volume (upper plot) and bulk modulus (lower plot) with increasing plane wave cutoff, as obtained from the equation of state of a $3 \times 3 \times 3$ cell of diamond. This equation of state was calculated at the MP2 level of theory in a cc-pVDZ pseudized Gaussian basis set, and fit to a Birch-Murnaghan form for 14 cell volumes at each plane wave truncation. ${ }^{46}$ 
approach to the basis construction for correlated calculations. In this, the relatively cheap mean-field part of the calculation can be performed with a plane wave basis, where convergence to the complete basis (e.g., to $1 \mathrm{meV}$ in energies) can be achieved using affordable energy cutoffs, and without the basis set linear-dependence that can be a problem in larger Gaussian basis sets. Once converged, this yields close to the optimal occupied orbitals, mean-field (Hartree-Fock) energy, and wavefunction, essentially free from basis set incompleteness or superposition errors. This is advantageous because the Gaussian basis will not in general span the full occupied orbital space, due to the polarization of the orbitals from the environment.

The large expansion of virtual orbitals as plane waves or virtual canonical orbitals is then avoided by representing the virtual space as an expansion of pseudized Gaussian basis functions, after having projected out the component of the (complete) occupied space from the Gaussian basis. This ensures that the full flexibility of the pseudized Gaussian basis is spanned, in addition to the complete occupied space. Since all orbitals are ultimately expressed by their Fourier components, this projection is trivially achieved, and the virtual orbital space $\left\{\left|\psi_{\alpha}\right\rangle\right\}$ can be constructed as

$$
\left|\psi_{\alpha}\right\rangle=\left|G_{\alpha}\right\rangle-\sum_{i}\left|\psi_{i}\right\rangle\left\langle\psi_{i} \mid G_{\alpha}\right\rangle,
$$

where $\left|G_{\alpha}\right\rangle$ is the Gaussian basis, and $\left|\psi_{i}\right\rangle$ represents the complete space of occupied orbitals expressed in the plane wave basis (note that the $\psi_{i}$ orbitals which are projected out refer to the "all-electron" orbitals rather than the pseudized orbitals to ensure true orthogonality). If the norm of any virtual orbital is below a threshold value after this projection, then it is removed from the calculation, while the rest are orthonormalized and constitute the virtual basis for the calculation. The virtual space is subsequently canonicalized before use in post-mean-field methods, with no further mixing between occupied and virtual states possible. It is also possible for the "occupied" atomic orbitals of the original contracted Gaussian basis to be identified and removed entirely from the basis, as they are largely redundant, to leave an overall basis the same size as the original, unmodified Gaussian basis, but still complete in all mean-field orbitals and properties.

The benefits of this basis construction are significant, with basis set incompleteness and superposition error only remaining in the correlated treatment of the wavefunction, which is readily extrapolatable within the employed correlation-consistent basis sets. A drawback of the above construction is that the occupied space is no longer represented within an underlying local basis, which may be desirable for the exploitation of locality approximations in quantum cluster methods. Finding a local representation would then require further localization steps which would be unnecessary if the underlying basis was already local. The use of Gaussian basis sets for local, cluster approximations will be explored in the future. We now turn to some applications to demonstrate the performance of the pseudized Gaussian basis compared to all-electron molecular calculations, before a study of more challenging systems.

\section{RESULTS}

\section{A. Comparison to molecular systems}

\section{He in a pseudized aug-cc-pVTZ basis set}

In order to quantify the effect of the pseudization, and to assess the fidelity of the representation of the Gaussian type orbitals, we first compare to gas phase molecular systems, where results within the same basis obtained from a molecular Gaussian basis code can be compared to our periodic implementation in the limit of a large simulation box. Here we use PSI $4^{47}$ as the molecular code while all pseudized GTO calculations were performed using VASP. The first investigation assesses the Hartree-Fock one-electron energies of a He atom in a $20 \times 20 \times 20 \AA^{3}$ cubic box. The He atom in the aug-cc-pvtz basis set constitutes a test case because it is free of frozen core states that might introduce an additional source of discrepancy between the VASP pseudopotential and PSI4 all-electron quantum chemistry results. For the sake of clarity we will refer to the results obtained using the VASP code and pseudized GTOs as PGTOs results, whereas the results obtained using PSI4 will be referred to as GTOs results. Figure 4 shows the Hartree-Fock (HF) one-electron energies calculated using $300 \mathrm{eV}, 600 \mathrm{eV}$, and $900 \mathrm{eV}$ plane wave cutoff energies. It can be seen that the $\mathrm{HF}$ one-electron energies converge rapidly for both occupied and virtual manifolds, even for the more high-lying states. It should be noted that both the virtual and occupied space was expressed in the underlying Gaussian basis in this example, rather than using the technique detailed in Section II D.

The order and degeneracy of the states agrees between the two different methods (PGTOs and GTOs). However, the inset in Figure 4 shows that the energy differences between the PGTOs and GTOs contain outliers corresponding to states 15 and 21-23, where the discrepancy can become larger than $4 \mathrm{eV}$. We attribute this to the different form of the PGTOs and the GTOs inside the PAW sphere. In this region the PGTOs are pseudized and augmented with terms that depend on the projectors, the all-electron partial waves, and the pseudoorbitals. However, we stress that the aim of this work is not

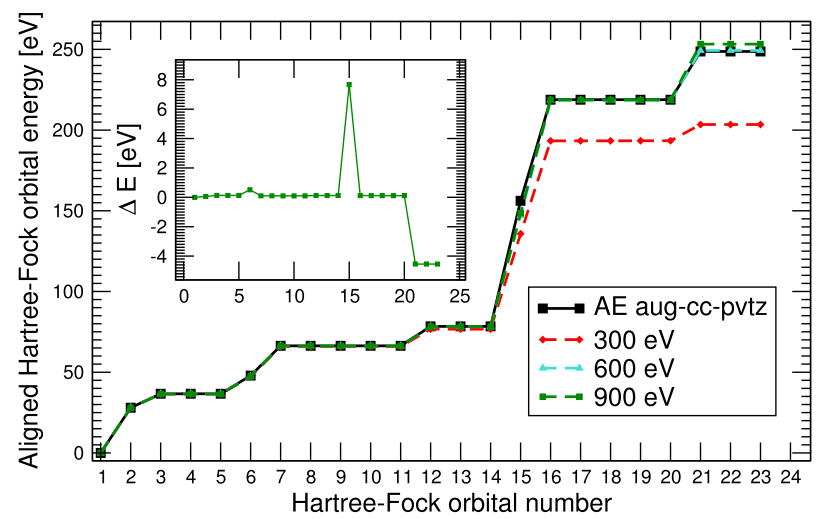

FIG. 4. Hartree-Fock (HF) one-electron energies retrieved as a function of the orbital number for the He atom. The all-electron (AE) HF orbital energies using the aug-cc-pVTZ basis set have been obtained using the PSI4 quantum chemistry package. The HF plane wave calculations using VASP have been performed in the same basis using a $20 \times 20 \times 20 \AA^{3}$ cubic box. 

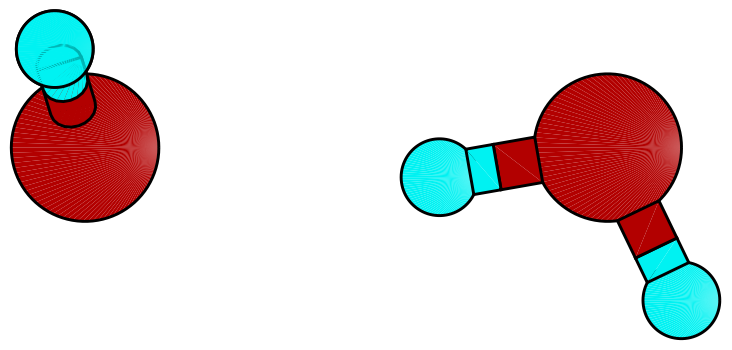

FIG. 5. Water dimer geometry.

to achieve perfect agreement between PGTOs and GTOs but rather to obtain a similar quality and basis set convergence for correlated wave function calculations.

\section{Water dimer}

Having demonstrated that the PGTOs yield a similar one-electron spectrum as the GTOs, as well as rapidly convergent properties with plane wave cutoff, we now turn to the discussion of the convergence of correlation energy contributions to the binding energy in the water dimer with basis size. Figure 5 and Table I show and specify the employed water dimer structure. This case is important because plane wave basis set calculations are computationally very expensive for atomic and molecular systems, which usually require a large simulation box with a lot of vacuum to minimize interactions between periodic images, and are required for many formation or cohesive energy calculations. As a result of the large box size the number of plane wave basis functions becomes very large regardless of the actual number of electrons in the unit cell. ${ }^{48}$ In this calculation we have employed a $10 \times 10 \times 10 \AA^{3}$ cubic box. The cutoff energy was set to $600 \mathrm{eV}$ resulting in a basis set size consisting of more than 30000 plane waves, which would be impossible to do at the level of MP2. However, the number of Gaussian basis functions is smaller than 200 even for the pseudized aug-cc-pVTZ basis set.

For this example (and subsequent applications in this work), the complete occupied space of states was first calculated and included within the basis to saturate the Hartree-Fock wavefunction. The PGTO basis was therefore only used to span the complementary virtual space, as described in Sec. II D. We note that the MP2 results for this system change by less than $3 \mathrm{meV}$ when employing a $15 \times 15 \times 15 \AA^{3}$ cubic box. This is unsurprising, since even the most diffuse oxygen functions in the aug-cc-pVTZ basis

TABLE I. Atomic positions for the water $\operatorname{dimer}^{\mathrm{a}}(\AA)$.

\begin{tabular}{cccr}
\hline \hline & $\mathrm{x}$ & $\mathrm{y}$ & \multicolumn{1}{c}{$\mathrm{z}$} \\
\hline $\mathrm{O}$ & 0.004 & 1.491 & 0.000 \\
$\mathrm{H}$ & 0.168 & 0.559 & 0.000 \\
$\mathrm{H}$ & 0.850 & 1.899 & 0.000 \\
$\mathrm{O}$ & 0.004 & -1.405 & 0.000 \\
$\mathrm{H}$ & -0.542 & -1.573 & 0.749 \\
$\mathrm{H}$ & -0.542 & -1.573 & -0.749 \\
\hline \hline
\end{tabular}

ahttp://cccbdb.nist.gov/. have less than $0.001 \%$ of their integrated radial density found more than $6 \AA$ away from their nuclear origin. This locality of basis coverage is the dominant factor in the compactness of the Gaussian basis for this purpose compared to plane wave expansions. We note here that in all calculations, a standard contracted Gaussian basis was used for the construction of the PGTOs.

Table II summarizes the obtained binding energies for the water dimer on the level of HF and MP2 theories calculated using the (pseudized) aug-cc-pVDZ and aug-cc-pVTZ contracted basis sets. The oxygen 1s orbitals of the GTO calculations were frozen to allow for fairer comparison to the valence-only PGTO calculations performed with the PAW method. Since the PGTOs are only used to span the virtual orbitals, the PGTOs Hartree-Fock contribution to the binding energies are independent of the basis set size, since these are necessarily complete and free of basis set errors. This also renders the occupied space and HF contribution free of basis set superposition error, as shown by the lack of a counterpoise correction to the values. It can be seen that the HF contribution from the true, gas-phase GTOs approaches that of the PGTOs as the basis is increased.

Table II shows that the PGTOs and GTOs yield MP2 binding energies that deviate by more than $20 \mathrm{meV}$ from each other when no allowance is made for basis set superposition error. However, the majority of this difference is due to the incompleteness in the HF contribution to the interaction in the GTO basis. The discrepancy in the MP2 correlation contribution to the interaction energy is only at most $6 \mathrm{meV}$, despite the contrasting occupied space. This difference decreases as the basis set is increased and the HF contribution of the GTO basis becomes increasingly complete to match the PGTO calculations. Basis set superposition errors are a wellknown drawback of atom-centered basis sets and can typically lead to an overestimation of binding energies. This is because more basis coverage is available for each monomer at shorter bond lengths, as it can exploit the basis coverage supplied by the overlap of the functions from the other monomer.

To better understand the origin of the difference between PGTOs and GTOs we have also included in Table II calculations with counterpoise corrections for the basis set superposition error (BSSE). Both our PGTO and GTO calculations lower the predicted binding energies by about $15 \mathrm{meV}$ and $20 \mathrm{meV}$ in the case of PGTOs and GTOs,

TABLE II. Binding energy contributions for the water dimer using HF and MP2 theories, comparing results from gas-phase calculations in a contracted aug-cc-pVXZ basis with frozen oxygen 1 s core, and our results from a periodic system in a $10 \times 10 \times 10 \AA^{3}$ cubic box, with a pseudized basis. The $(C P)$ denotes that counterpoise corrections have been included for basis set superposition error in this basis. All units are in $\mathrm{meV}$.

\begin{tabular}{lccccccc}
\hline \hline & \multicolumn{3}{c}{ PGTOs } & & \multicolumn{3}{c}{ GTOs } \\
\cline { 2 - 4 } \cline { 7 - 8 } Basis & HF & MP2 corr. & MP2 & & HF & MP2 corr. & MP2 \\
\hline aVDZ & 142 & 56 & 197 & & 154.9 & 62.4 & 217.3 \\
aVTZ & 142 & 66 & 208 & & 145.4 & 68.6 & 214.9 \\
aVDZ (CP) & 142 & 41 & 183 & & 151.2 & 42.5 & 193.7 \\
aVTZ (CP) & 142 & 53 & 195 & 143.8 & 52.8 & 196.6 \\
\hline \hline
\end{tabular}


respectively, if BSSE is accounted for. This basis set superposition error in the case of the PGTO is purely contained in the correlation part of the MP2 since the occupied space is complete, while for the GTO basis it also includes basis set superposition in the HF contribution. Once the BSSE of purely the correlation part of the MP2 interaction energy is analysed, the errors are in closer agreement, however, these will still be affected by the contrasting occupied space in each system. From this example we conclude that our PGTO basis sets yield a comparably accurate description of electronic correlation effects as the GTO counterparts for molecular systems. We note that the achieved accuracy of a few meV provides confidence in the constructed basis, and is sufficiently accurate to allow for reliable predictions in ab initio calculations.

\section{Nitrogen molecule}

As a final comparison for molecular systems, we now seek to investigate the performance of the pseudized GTO basis for the atomization of a prototypical covalently bound system, the nitrogen molecule. The calculations of $\mathrm{N}_{2}$ were performed using a $15 \times 15 \times 15 \AA^{3}$ cubic unit cell to minimize the interaction between the periodic images. The plane wave energy cutoff was set to $600 \mathrm{eV}$. A bond length of $1.0656 \AA$ was used as the equilibrium geometry.

Table III summarizes the obtained binding energies on the level of HF and MP2 theories using PGTOs and GTOs, with and without counterpoise corrections for the BSSEs, with $\mathrm{UHF} / \mathrm{UMP} 2$ used for the calculation of the atomic system in each instance. Again our results show that the PGTO Hartree-Fock contributions to the dissociation energy are independent of basis size and agree well with the GTO results from large basis sets. The GTO results using aug-cc-pVQZ yield $\mathrm{HF}$ atomization energies that deviate by less than $2 \mathrm{meV}$ from our results obtained using PGTOs within VASP. On the level of MP2 theory the PGTOs and GTOs results differ more strongly, especially for the aVDZ basis set. This is a result of the number of differences: the PAW framework for the core electrons, the pseudization of the basis, and the different virtual space, due to the construction detailed in Section II D and orthogonalization to the complete occupied

TABLE III. Binding energy contributions for the nitrogen molecule using HF and MP2 theories, for all-electron, gas-phase GTO results, and our contracted PGTO implementation. The first column defines the type of contracted aug-cc-pVXZ basis set employed. $(C P)$ denotes that the results have been corrected for basis set superposition error via counterpoise correction. All units are in $\mathrm{eV}$.

\begin{tabular}{lccccccr}
\hline \hline & \multicolumn{3}{c}{ PGTOs } & & \multicolumn{3}{c}{ GTOs } \\
\cline { 2 - 4 } \cline { 6 - 8 } Basis & HF & MP2 corr. & MP2 & & HF & MP2 corr. & MP2 \\
\hline aVDZ & 5.094 & 4.153 & 9.247 & 4.754 & 4.335 & 9.089 \\
aVTZ & 5.094 & 4.771 & 9.864 & 5.043 & 5.046 & 10.089 \\
aVQZ & 5.094 & $\ldots$ & $\ldots$ & 5.096 & 5.111 & 10.207 \\
aVDZ $(\mathrm{CP})$ & 5.094 & 3.944 & 9.038 & 4.722 & 4.157 & 8.879 \\
aVTZ (CP) & 5.094 & 4.674 & 9.768 & 5.033 & 4.731 & 9.764 \\
aVQZ (CP) & 5.094 & $\ldots$ & $\ldots$ & 5.094 & 5.004 & 10.097 \\
\hline \hline
\end{tabular}

space. However, as the basis is increased to aug-cc-pVTZ, the agreement is improved, likely to be due to the fact that the larger space mitigates the discrepancies in the construction of the virtual space, as the occupied space of the GTOs is rapidly approaching completeness. Once corrections for BSSE are also included, the agreement in the MP2 is very good, with the PGTOs and GTOs predicting a correlation energy contribution of $4.674 \mathrm{eV}$ and $4.731 \mathrm{eV}$ to the binding energy, respectively.

However, the correlation energy contribution to the binding energy converges very slowly with respect to the largest angular momentum quantum number included in the basis, and comparison to the aug-cc-VQZ GTO basis results shows that we are not yet converged to "chemical accuracy." Since we have not yet implemented the required transformation routines for $g$-functions, we cannot employ aug-cc-pVQZ PGTO basis sets or larger for this element. We also stress that explicitly correlated methods will greatly help in yielding a more rapid convergence of the binding energies with respect to the basis set size for such covalently bonded systems. These methods and their implementations are already being investigated in the framework of fully periodic systems using a plane wave basis set and the combination of these different techniques will be subject to a future study.,49 Nonetheless our results indicate that the pseudized GTOs yield results that are very similar compared to the all-electron GTO results. While establishing the validity of this comparison is important, the aim of this procedure is not for application to molecular systems, but rather for a compact basis for extended systems, which we now consider and compare to experiment.

\section{B. Extended systems}

\section{Neon solid}

One area where a local Gaussian virtual space representation is expected to perform well compared to plane wave expansions is in the description of weakly interacting, dispersion dominated extended systems. The contracted aug-cc-pVXZ hierarchy is expected to provide a rapidly convergent and systematic truncation of the virtual basis by spanning a space constructed to obtain the required higher energy excitations for the dispersion interaction, as compared to a strict energetic truncation of plane wave or canonical virtual orbitals. This systematic truncation can be of great benefit if one seeks to calculate converged energy differences between solids and isolated atoms, where a strict orbital energetic truncation becomes physically meaningless. To this end, we demonstrate the calculation of the atomization energy of the neon noble gas solid. The neon solid has an fcc unit cell, with a lattice constant of $4.641 \AA$, and the pseudized Gaussian basis for the virtual space is expanded in plane waves up to a cutoff parameter of $700 \mathrm{eV}$. The MP2 results of the solid have been calculated using a $6 \times 6 \times 6 k$-point mesh, while the box size for the atomic system is $30 \times 30 \times 30 \AA^{3}$. For the HF contribution to the cohesive energy we choose an even denser $k$-point mesh of $14 \times 14 \times 14$. These parameters are sufficient to converge the cohesive energy to within $1 \mathrm{meV}$. 
TABLE IV. Cohesive energy of the neon solid using HF and MP2 theories, in PGTO basis sets, with and without corrections for BSSE. Comparison to the incremental results of Ref. 50 for the MP2 energy are included. All units are in meV.

\begin{tabular}{lcc}
\hline \hline Basis & HF & MP2 \\
\hline aug-cc-pVDZ (no CP) & -8 & 19 \\
aug-cc-pVTZ (no CP) & -8 & 24 \\
aug-cc-pVDZ (CP) & -8 & 10 \\
aug-cc-pVTZ (CP) & -8 & 16 \\
aug-cc-pV(D,T)Z (extrap,CP) & -8 & 19 \\
Incremental method & & 18.8 \\
\hline \hline
\end{tabular}

Results for the atomization energy of this system can be seen in Table IV, where we present HF and MP2 results obtained using PGTOs with and without counterpoise (CP) corrections for the BSSE. Since the occupied space is the same for each basis, as the basis choice simply affects the virtual orbitals in this scheme, the Hartree-Fock contribution to the atomization energy in each basis is also seen to be the same. The binding of the solid is also purely dispersive. As dispersive interactions are a manifestation of correlated phenomena, this renders the Hartree-Fock contribution negative, representing a repulsive interaction at this level of theory. We therefore consider the atomization energy at the level of MP2 theory (which is believed to describe dispersion interactions well in this system) with different choices of virtual basis.

The benchmark MP2 result for this system has been obtained using the incremental method, as detailed in Ref. 50, which relies on a truncated many-body expansion for the interactions, and has been shown to work particularly well for such noble gas solids or molecular crystals. ${ }^{51}$ Our findings indicate again that BSSEs can be quite large on a relative scale for these weakly bound systems and need to be accounted for. If CP corrections are included, our aug-cc-pVDZ and aug-cc-pVTZ PGTO calculations predict MP2 atomization energies of $10 \mathrm{meV}$ and $16 \mathrm{meV}$, respectively. To correct for the remaining basis set incompleteness error we can also perform a complete basis set (CBS) limit extrapolation, theoretically justified according to the $1 / L^{3}$ convergence behavior of the correlation energy. ${ }^{39,52,53}$ The obtained CBS limit result is $19 \mathrm{meV}$, which is in good agreement with results obtained using the incremental method of $18.8 \mathrm{meV}$, although we believe that the remaining error from $k$-point sampling and convergence of other technical parameters in our calculations is on the order of $1 \mathrm{meV}$, and therefore such good agreement is somewhat fortuitous. We note that the experimental value of the atomization energy corrected for zero-point vibrational effects is $27.8 \mathrm{meV},{ }^{50,54-56}$ demonstrating an underbinding of MP2, consistent with the trend observed for gas-phase noble gas dimers and of previous MP2 results for the neon crystal. $^{50,57-59}$

\section{Water at LiH}

As a final application, we study water adsorption onto the surface of a lithium hydride crystal at the level of MP2 theory. Although dissolution is the fate of this ionic crystal

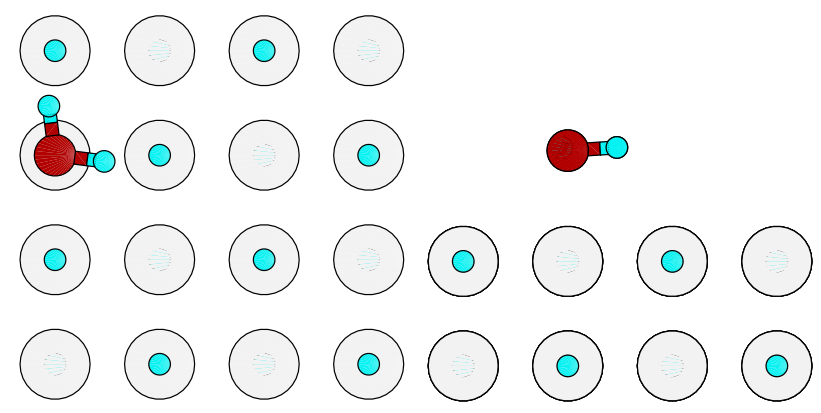

FIG. 6. H2O@LiH adsorption site studied.

upon solvation, this process is first instigated by the adsorption of a single water molecule, and the system has been studied extensively and with high accuracy by incremental methods and diffusion Monte Carlo. ${ }^{60,61}$ In contrast to these, our work employs fully periodic boundary conditions, and the projected Gaussian space for the virtuals is expected to be efficient in these cases since there is much vacuum required in the simulation cell to avoid spurious periodic images. ${ }^{48}$ Figure 6 shows the relaxed structure of the adsorbed water molecule on the $\mathrm{LiH}$ crystal. The structures have been relaxed using the DFT-PBE functional. ${ }^{62}$ Only the atoms of the water molecule have been allowed to relax. The $\mathrm{LiH}$ surface is modelled using a two-layer surface supercell containing $16 \mathrm{Li}$ and $16 \mathrm{H}$ atoms. These atoms have been kept fixed to the $\mathrm{LiH}$ crystal atom positions with a lattice constant corresponding to $4.1108 \AA$ A. The O $1 s$ states have been kept frozen in the MP2 calculation. All other electronic states have been treated as valence states.

Table V summarizes the binding energies of the water molecule for different methods. The DFT-PBE functional yields a binding energy of $214 \mathrm{meV}$, which agrees well with the value of $212 \mathrm{meV}$ reported in Ref. 60. On the level of Hartree-Fock, the water molecule exhibits a binding energy of $44 \mathrm{meV}$ for the relaxed structures. However, adding the electron correlation effects on the level of MP2 theory yields an adsorption energy for water of $157 \mathrm{meV}$ and $195 \mathrm{meV}$ for the pseudized cc-pVDZ and cc-pVTZ basis sets, respectively. The MP2 calculations include CP corrections for the BSSE. A simple CBS limit extrapolation yields an adsorption energy of $211 \mathrm{meV}$.

TABLE V. Adsorption energies for one water molecule on the $\mathrm{LiH}$ surface at various levels of theory. Hartree-Fock and MP2 results are performed in different PGTO basis sets, corrected for BSSE, and extrapolated to the CBS limit for comparison. Results from the incremental method and DMC are taken from Ref. 60. All units are in meV.

\begin{tabular}{lrrr}
\hline \hline & \multicolumn{3}{c}{$E_{\text {ads }} / \mathrm{meV}$} \\
\cline { 2 - 4 } & cc-pVDZ & cc-pVTZ & CBS \\
\hline HF & 44 & 44 & 44 \\
MP2 & 157 & 195 & 211 \\
\hline DFT-PBE & & & 214 \\
Incremental [CCSD(T)] & & & 246 \\
DMC & & & 237 \\
\hline \hline
\end{tabular}


TABLE VI. Adsorption energies for one water molecule on the LiH surface using MP2 theory employing different virtual orbital manifolds with and without counterpoise corrections for the BSSE. cc-pV(D,T)Z denotes the three-point extrapolation to the complete basis limit. ${ }^{53}$ All units are in $\mathrm{meV}$.

\begin{tabular}{lccc}
\hline \hline Basis set & $N_{\mathrm{v}}$ & $E_{\text {ads }}^{\mathrm{MP2}}$ & $E_{\text {ads }}^{\mathrm{MP}(\mathrm{CP})}$ \\
\hline cc-pVDZ & 328 & 510 & 157 \\
cc-pVTZ & 762 & 365 & 195 \\
cc-pV(D,T)Z & & 304 & 211 \\
Canonical HFOs & 20523 & 217 & 217 \\
\hline \hline
\end{tabular}

To further verify the PGTOs approach we have also performed a calculation with the full set of canonical Hartree-Fock orbitals constructed from diagonalization of the Fock operator in the complete plane wave basis set. We note that this approach additionally employs a basis set extrapolation technique which is outlined in Ref. 6. The obtained adsorption energy of $217 \mathrm{meV}$ is in very good agreement with the other complete basis set limit findings within the far smaller PGTO basis sets, summarized in Table VI. However, if this huge set of canonical virtual orbitals is truncated to similar sizes as the PGTO virtual space, then results are poor, and comparison to the bulk or isolated molecule is difficult, as it is hard to truncate the canonical space consistently for the different systems. It is remarkable to see that PGTOs and complete canonical HF orbitals yield results that agree to within $6 \mathrm{meV}$. However, we stress again that it is extremely important to correct for BSSEs in the calculations as can be seen by comparing $E_{\mathrm{ads}}^{\mathrm{MP} 2}$ and $E_{\mathrm{ads}}^{\mathrm{MP2}(\mathrm{CP})}$ in Table VI, where the BSSE is larger than the binding energy itself. Furthermore Table VI also presents the number of virtual orbitals employed in the different calculations. The biggest basis set in the PGTOs and the full plane wave basis in the canonical HFOs calculations corresponds to 762 and 20523 orbitals, respectively. This comparison demonstrates strongly how much more compact the PGTOs basis for such systems can become compared to canonical HF orbitals. In a future study we will investigate this system in greater detail including methods that also go beyond MP2 theory, as correlations beyond this level are clearly important, as can be seen by comparison to the $\operatorname{CCSD}(\mathrm{T})$ incremental results, and diffusion Monte Carlo.

\section{CONCLUSION}

In this paper we have outlined a simple, black-box, and robust approach to use local, atom-centered Gaussian basis functions within a plane wave basis set using the projector augmented wave method and periodic boundary conditions. The so-called pseudized Gaussian basis set can be expanded efficiently in a plane wave basis set with a moderate kinetic energy cutoff. We have shown that a hybrid approach whereby the occupied orbitals are expanded in a plane wave basis set and only the virtual orbital manifold is expanded in the pseudized Gaussian basis set orthogonalized to the occupied orbitals provides a compact and systematically improvable basis. The advantages of this over pure plane wave basis set calculations become most beneficial in correlated wavefunction based calculations of low dimensional systems and systems where weak interactions need to be described with high accuracy. This is not unexpected since the size of the plane wave basis set suffers from the fact that it grows linearly with the box size regardless of the position or number of atoms in the studied system. As a result it is difficult to devise systematic virtual orbital manifold truncation schemes for rapidly convergent energy differences. In contrast to plane waves, Gaussian atom-centered basis sets take the local character of electronic correlation into account a priori and allow for a systematic description of electronic correlation effects such as the polarizability using a system tailored and compact basis set.

Of course, the introduction of such local basis sets also always bears the burden of several shortcomings such as basis set superposition errors (BSSE) and linear dependencies of diffuse atom-centered basis functions in densely packed solids. These problems can partly be accounted for by counterpoise BSSE corrections and removing linearly dependent basis functions. The compromise of a wavefunction expansion in Gaussians for the virtual space and a plane wave expansion for the occupied space seems an efficient approach for combining the advantages and mitigating the disadvantages of each basis. We note that our method allows to easily switch between these two different basis sets (local atomcentered Gaussians and periodic plane wave), which could potentially lead to novel, transferable, and more compact basis sets with the aim to reduce the computational cost of correlated wavefunction based theories in periodic systems even further. In the future, this infrastructure will be combined with other correlated methods, including coupled-cluster and F12 methods. ${ }^{4,8,9,49,63,64}$ Furthermore, methods which directly exploit the locality of correlation effects, including quantum cluster methods such as dynamical mean-field theory ${ }^{65}$ and density matrix embedding theory, ${ }^{66-69}$ as well as more traditional domain-based approaches to local correlation ${ }^{25,36,70}$ can be used within this framework, and are being actively explored.

\section{ACKNOWLEDGMENTS}

We gratefully acknowledge the help with the implementation in VASP and useful discussions with Georg Kresse and Martijn Marsman. G.H.B gratefully acknowledges funding from the Royal Society. G. K.-L. Chan acknowledges support from the US Department of Energy through Grant No. DE-SC0010530, with secondary support from Grant No. DE-SC0008624 (SciDAC).

${ }^{1}$ T. Helgaker, P. Jørgensen, and J. Olsen, Molecular Electronic-Structure Theory (Wiley, 2000).

${ }^{2}$ J. J. Shepherd, A. Grüneis, G. H. Booth, G. Kresse, and A. Alavi, Phys. Rev. B 86, 1-14 (2012), e-print arXiv:1202.4990.

${ }^{3}$ J. J. Shepherd, G. H. Booth, and A. Alavi, J. Chem. Phys. 136, 244101 (2012).

${ }^{4}$ A. Grüneis, J. J. Shepherd, A. Alavi, D. P. Tew, and G. H. Booth, J. Chem. Phys. 139, 084112 (2013).

5J. J. Shepherd and A. Grüneis, Phys. Rev. Lett. 110, 1-5 (2013), e-print arXiv: 1310.6059.

${ }^{6}$ M. Marsman, A. Grüneis, J. Paier, and G. Kresse, J. Chem. Phys. 130, 184103 (2009).

${ }^{7}$ A. Gulans, J. Chem. Phys. 141, 164127 (2014). 
${ }^{8}$ A. Grüneis, G. H. Booth, M. Marsman, J. Spencer, A. Alavi, and G. Kresse, J. Chem. Theory Comput. 7, 2780-2785 (2011).

${ }^{9}$ G. H. Booth, A. Gruneis, G. Kresse, and A. Alavi, Nature 493, 365-370 (2013).

${ }^{10}$ F. Ma, W. Purwanto, S. Zhang, and H. Krakauer, Phys. Rev. Lett. 114, 226401 (2015).

${ }^{11}$ B. D. Dunnington and J. R. Schmidt, J. Chem. Phys. 143, 104109 (2015).

${ }^{12}$ J. I. Mustafa, S. Coh, M. L. Cohen, and S. G. Louie, Phys. Rev. B 92, 165134 (2015).

${ }^{13}$ O. K. Andersen, Phys. Rev. B 12, 3060 (1975).

${ }^{14}$ T. Kotani and M. van Schilfgaarde, Phys. Rev. B 81, 125117 (2010).

${ }^{15}$ A. Gulans, S. Kontur, C. Meisenbichler, D. Nabok, P. Pavone, S. Rigamonti, S. Sagmeister, U. Werner, and C. Draxl, J. Phys.: Condens. Matter 26, 363202 (2014).

${ }^{16}$ L. Genovese, A. Neelov, S. Goedecker, T. Deutsch, S. A. Ghasemi, A. Willand, D. Caliste, O. Zilberberg, M. Rayson, A. Bergman, and R. Schneider, J. Chem. Phys. 129, 014109 (2008).

${ }^{17}$ T. Yanai, G. I. Fann, Z. Gan, R. J. Harrison, and G. Beylkin, J. Chem. Phys. 121, 2866-2876 (2004).

${ }^{18}$ T. A. Arias, Rev. Mod. Phys. 71, 267-311 (1999).

${ }^{19}$ V. Blum, R. Gehrke, F. Hanke, P. Havu, V. Havu, X. Ren, K. Reuter, and M. Scheffler, Comput. Phys. Commun. 180, 2175-2196 (2009).

${ }^{20}$ J. M. Soler, E. Artacho, J. D. Gale, A. Garca, J. Junquera, P. Ordejn, and D. Snchez-Portal, J. Phys.: Condens. Matter 14, 2745-2779 (2002).

${ }^{21}$ T. H. Dunning, J. Chem. Phys. 90, 1007-1023 (1989).

${ }^{22}$ N. Balabanov and K. Peterson, J. Chem. Phys. 123, 064107 (2005).

${ }^{23}$ R. Dovesi, R. Orlando, A. Erba, C. M. Zicovich-Wilson, B. Civalleri, S. Casassa, L. Maschio, M. Ferrabone, M. De La Pierre, P. DArco, Y. Noel, M. Causa, M. Rerat, and B. Kirtman, Int. J. Quantum Chem. 114, 1287-1317 (2014).

${ }^{24}$ C. Pisani, M. Schtz, S. Casassa, D. Usvyat, L. Maschio, M. Lorenz, and A. Erba, Phys. Chem. Chem. Phys. 14, 7615 (2012).

${ }^{25}$ L. Maschio, D. Usvyat, M. Schtz, and B. Civalleri, J. Chem. Phys. 132, 134706 (2010).

${ }^{26}$ M. V. Arnim and R. Ahlrichs, J. Comput. Chem. 19, 1746 (1998).

${ }^{27}$ G. Lippert, J. Hutter, and M. Parrinello, Theor. Chem. Acc. 103, 124 (1999).

${ }^{28}$ M. Krack and M. Parrinello, Phys. Chem. Chem. Phys. 2, 2105 (2000).

${ }^{29}$ K. N. Kudin and G. E. Scuseria, Phys. Rev. B 61, 16440 (2000).

${ }^{30}$ L. Füsti-Molnár and P. Pulay, J. Chem. Phys. 117, 7827 (2002).

${ }^{31}$ J. VandeVondele and J. Hutter, J. Chem. Phys. 118, 4365 (2003).

${ }^{32}$ S. Goedecker, Rev. Mod. Phys. 71, 1085-1123 (1999).

${ }^{33}$ M. C. Strain, G. E. Scuseria, and M. J. Frisch, Science 271, 51-53 (1996).

${ }^{34}$ J. C. Burant, G. E. Scuseria, and M. J. Frisch, J. Chem. Phys. 105, 8969-8972 (1996).

${ }^{35}$ S. Goedecker and G. E. Scuseria, Comput. Sci. Eng. 5, 14-21 (2003).

${ }^{36}$ C. Pisani, L. Maschio, S. Casassa, M. Halo, M. Schtz, and D. Usvyat, J. Comput. Chem. 29, 2113-2124 (2008).

${ }^{37}$ M. Del Ben, J. Hutter, and J. VandeVondele, J. Chem. Theory Comput. 8, 4177 (2012).
${ }^{38}$ T. Kato, Commun. Pure Appl. Math. 10, 151-177 (1957).

${ }^{39}$ W. Kutzelnigg and J. D. Morgan III, J. Chem. Phys. 96, 4484 (1992).

${ }^{40}$ G. H. Booth, D. Cleland, A. Alavi, and D. P. Tew, J. Chem. Phys. 137, 164112 (2012).

${ }^{41}$ P. Pulay, Chem. Phys. Lett. 100, 151 (1983).

${ }^{42}$ P. E. Blöchl, Phys. Rev. B 50, 17953-17979 (1994).

${ }^{43}$ G. Kresse and D. Joubert, Phys. Rev. B 59, 1758-1775 (1999).

${ }^{44} \mathrm{G}$. Kresse and J. Hafner, J. Phys.: Condens. Matter 6, 8245-8257 (1994).

${ }^{45}$ F. Gygi and A. Baldereschi, Phys. Rev. B 34, 4405 (1986).

${ }^{46}$ F. Birch, Phys. Rev. 71, 809-824 (1947).

${ }^{47}$ J. M. Turney, A. C. Simmonett, R. M. Parrish, E. G. Hohenstein, F. Evangelista, J. T. Fermann, B. J. Mintz, L. A. Burns, J. J. Wilke, M. L. Abrams, N. J. Russ, M. L. Leininger, C. L. Janssen, E. T. Seidl, W. D. Allen, H. L. Schaefer, R. A. King, E. F. Valeev, C. D. Sherrill, and T. D. Crawford, Wiley Interdiscip. Rev.: Comput. Mol. Sci. 2, 556 (2012).

${ }^{48}$ M. Preuss, W. G. Schmidt, K. Seino, J. Furthmuller, and F. Bechstedt, J. Comput. Chem. 25, 112 (2004).

${ }^{49}$ A. Grüneis, Phys. Rev. Lett. 115, 066402 (2015).

${ }^{50}$ P. Schwerdtfeger, B. Assadollahzadeh, and A. Hermann, Phys. Rev. B 82, $1-11(2010)$

${ }^{51}$ B. Paulus, Phys. Rep. 428, 1 (2006).

${ }^{52}$ W. Kutzelnigg and J. D. Morgan III, J. Chem. Phys. 97, 8821 (1992).

${ }^{53}$ T. Helgaker, W. Klopper, H. Koch, and J. Noga, J. Chem. Phys. 106, 9639 (1997).

${ }^{54}$ D. N. Batchelder, D. L. Losee, and R. O. Simmons, Phys. Rev. 162, 767 (1967).

${ }^{55}$ D. N. Batchelder, D. L. Losee, and R. O. Simmons, Phys. Rev. 173, 873 (1968).

${ }^{56}$ Y. Endoh, G. Shirane, and J. Skalyo, Jr., Phys. Rev. B 11, 1681 (1975).

${ }^{57}$ A. West and F. Merkt, J. Chem. Phys. 118, 8807 (2003).

${ }^{58}$ J. F. Ogilvie and F. Y. H. Wang, J. Mol. Struct. 273, 277 (1992).

${ }^{59}$ P. R. Herman, P. E. LaRoque, and B. P. Stoicheff, J. Chem. Phys. 89, 4535 (1988).

${ }^{60}$ S. Binnie, "Ab initio surface energetics: Beyond chemical accuracy," Ph.D. Thesis (University College, London, 2011).

${ }^{61}$ S. J. Binnie, S. J. Nolan, N. D. Drummond, D. Alfè, N. L. Allan, F. R. Manby, and M. J. Gillan, Phys. Rev. B 82, 165431 (2010).

${ }^{62}$ J. P. Perdew, K. Burke, and M. Ernzerhof, Phys. Rev. Lett. 77, 3865-3868 (1996).

${ }^{63}$ A. Gruneis, M. Marsman, J. Harl, L. Schimka, and G. Kresse, J. Chem. Phys. 131, 154115 (2009).

${ }^{64}$ D. Usvyat, J. Chem. Phys. 139, 194101 (2013).

${ }^{65}$ G. Kotliar, S. Y. Savrasov, K. Haule, V. S. Oudovenko, O. Parcollet, and C. A. Marianetti, Rev. Mod. Phys. 78, 865-951 (2006).

${ }^{66}$ G. Knizia and G. K.-L. Chan, Phys. Rev. Lett. 109, 186404 (2012).

${ }^{67}$ B.-X. Zheng and G. K.-L. Chan, Phys. Rev. B 93, 035126 (2016).

${ }^{68}$ I. W. Bulik, W. Chen, and G. E. Scuseria, J. Chem. Phys. 141, 054113 (2014).

${ }^{69}$ G. H. Booth and G. K.-L. Chan, Phys. Rev. B 91, 155107 (2015).

${ }^{70}$ M. Schwilk, D. Usvyat, and H.-J. Werner, J. Chem. Phys. 142, 121102 (2015). 